\title{
Cáncer de vejiga en hospitales públicos de Madrid
}

\author{
Cabrera Cabrera JA. ${ }^{1}$, Cabrera López I. ${ }^{2}$, Domínguez Gordillo A. ${ }^{3}$, Hernando Arteche A. ${ }^{4}$, \\ Alpuente Román JC. ${ }^{4}$, Méndez Montesino JR. ${ }^{5}$, Moreno Reyes A. ${ }^{6}$
}

Sanid. mil. 2014; 70 (3): 197-203; ISSN: 1887-8571

\begin{abstract}
RESUMEN
Con diseño retrospectivo se analizan 4.452 pacientes con cáncer de vejiga, procedentes del Registro de Tumores de Madrid. La serie ha sido seguida durante 15 años. Se estudian las variables que dicho registro posee. La edad media ha sido de 68,7 (DT 11,95). La razón de sexo ha sido de 5:1. Se ha encontrado la siguiente histología: N. Uroteliales 94,3\%, N. Células escawmosas 3,2\%, N. glandulares $0,9 \%$ y otras histologías $0,5 \%$. La extensión tumoral más frecuente se ha correspondido con la localizada con un $83,5 \%$, seguida de la regional con un 10,6\%, la diseminada con un 4,9\% y por último los "in situ" con un 0,7\%. Los tratamientos han sido: Cirugía 37,8\%; Cirugía más radioterapia 6,2\%; Cirugía más Quimioterapia 38,4\%; Cirugía más Inmunoterapia 6\% y paliativos 2,7\%. Se ha encontrado un $4 \%$ de tumores primarios múltiples. Este estudio ha permitido conocer la realidad del cáncer vesical en todas sus vertientes por proceder los pacientes del día a día de la misma población en la que se diagnosticaron a los enfermos.
\end{abstract}

PALABRAS CLAVE: Cáncer de vejiga, Epidemiología.

\section{Bladder Cancer in public hospitals in Madrid}

SUMMARY: Retrospective design with 4,452 patients with bladder cancer from the Cancer Registry of Madrid are analyzed. The series has been followed for 15 years. Variables such registration has studied. The mean age was 68.7 (SD 11.95). The sex ratio was 5:1. We found the following histology: N. urothelial $94.3 \%$, N. squamous cells $3.2 \%$, N. glandular histologies $0.9 \%$ and other $0.5 \%$. The most frequent tumor extension has corresponded with localized with $83.5 \%$, followed by $10.6 \%$ with regional, disseminated with $4.9 \%$ and finally the "in situ" with $0.7 \%$. The treatments were: surgery $37.8 \%$; surgery and radiotherapy $6.2 \%$; surgery and chemotherapy $38.4 \%$; immunotherapy and surgery $6 \%$ and palliative $2.7 \%$. Found $4 \%$ of multiple primary tumors. This study has shown the reality of bladder cancer in all its aspects by patients come daily from the same population in which the patients were diagnosed.

KEY WORDS: Bladder cancer, Epidemiology.

\section{INTRODUCCION}

En 2.012, Eucan publica un total de 151.189 casos nuevos, con 52.358 fallecimientos; en España y para el mismo año encuentra 13.789 casos con 5.007 defunciones; por sexos y referidos a nuestro País la distribución ha sido de 11.584 varones y 2.205 mujeres. A la vista de estos resultados podemos decir que aporta a la enfermedad cancerosa un 6,4\% ${ }^{1}$. La mortalidad encontrada por el Centro Nacional de Epidemiología del Instituto de Salud Carlos III, ha sido en el año 2011 para España y en varones del 12,81 por cien mil, destacándose Melilla con un 27,33 y Guadalajara con un 3,5. Madrid está un poco por debajo de la media nacional, 12,5 por cien mil; en el caso de las mujeres

1 Médico Urólogo. Hospital La Milagrosa. Servicio de Urología. Madrid. España.

2 MIR. Hospital Niño Jesús. Servicio de Pediatría. Madrid. España.

${ }^{3}$ Universidad Complutense de Madrid. Facultad de Medicina. Departamento de Medicina Preventiva, Salud Pública y Comunitaria e $\mathrm{H}^{\mathrm{a}}$ de la Ciencia. Madrid. España.

${ }^{4}$ Médico civil. Hospital Central de la Defensa Gómez Ulla. Servicio de Urología. Madrid. España.

${ }^{5}$ Cor. Médico. Hospital Central de la Defensa Gómez Ulla. Servicio de Medicina Preventiva. Madrid. España.

${ }^{6}$ Tcol. Médico. Hospital Central de la Defensa Gómez Ulla. Servicio de Urología. Madrid. España.

Dirección para correspondencia: urojacc82@yahoo.es

Recibido: 19 de septiembre de 2013

Aceptado: 5 de noviembre de 2013 para España se ofrece una tasa de 1,95 por cien mil, los extremos han correspondido en este caso a León con un 3,53 y a Melilla con un 0 por cien mil, situándose Madrid en el 2,2 por cien mil².

\section{OBJETIVOS}

Conocer las caracteristicas de persona y del tumor en la población que es asistida en los Hospitales públicos de Madrid, que son los que van a acudir a nuestro Hospital. Analizar las posibles asociaciones entre las diferentes variables.

\section{SUJETOS Y MÉTODOS}

Se trata de un estudio retrospectivo. Los pacientes proceden del Registro de Tumores de la Comunidad de Madrid, quien recoge esta patología de los Hospitales Públicos radicados en la misma. Consta de 4.452 pacientes diagnosticados 1 de enero de 1990 y 31 de diciembre de 2004. La distribución cronológica se ha agrupado en quinquenios. Se estudian las variables de persona, las correspondientes al tumor y a su tratamiento. La edad se ha agrupado en sujetos menores de 70 años y de 70 y más, tomando como cifra de corte la edad media de la serie. La histología se ha agrupado en Neoplasias uroteliales, Neoplasias de células escamosas, Neoplasias glandulares, Otras neoplasias 
y Desconocido. Para la extensión tumoral, se ha empleado la clasificación del Surveillance, Epidemiology and End Results (SEER), Programa modificado por la American Joint Commitee Cancer (AJCC) $)^{3}$. Los tratamientos se han agrupado en: Sin tratamiento (entendiendo por tal los que se han tratado en otros hospitales), Cirugía, Paliativos, Cirugía más Radioterapia, Cirugía más quimioterapia, Cirugía más inmunoterapia y otros. Los tumores primarios múltiples se han definido de acuerdo con la IARC ${ }^{4}$. En el análisis estadístico se ha utilizado el paquete SPSS 15.0 para Windows. Se han cruzado todas las variables entre sí, empleandose el chi cuadrado para establecer posibles asociaciones, trabajando en todo momento con una $\mathrm{p}$ $\leq 0,05$.

\section{RESULTADOS}

El total de la población estudiada son 4.452 sujetos diagnosticados de cáncer de vejiga entre 1 de enero de 1990 y 31 de diciembre de 2004, recogidos de la base de datos del Registro Central de Tumores de la Comunidad de Madrid. La distribución de los casos a lo largo del periodo de estudio aumentó especialmente en el quinquenio 1995-1999, disminuyendo en el siguiente (Tabla 1).

Del total de casos, 3.711 eran varones, comentando que se encontró un hermafrodita verdadero, que para evitar distorsiones ulteriores lo hemos considerado como perdido por el sistema. El numero de casos fue sensiblemente superior entre los varones $(83,4 \%$ vs $16,6 \%)$, manteniendose esta diferencia a lo largo del periodo de estudio. La edad de nuestros pacientes estaba comprendida entre menores de un año y los 102, con una media de 68,87 (DT de 11,95) y una mediana de 69,99.

La edad media en los varones ha sido de 68,5 años (DT 11,4) y en las mujeres de 70,5 años (DT 14,2).

Por sexo y grupos de edad ( $<70$ años $y \geq 70$ años), se han encontrado diferencias estadísticamente significativas $(\mathrm{p}<0,001)$ tanto entre los menores y mayores de 70 años con respecto al sexo. En los hombres el $55,1 \%$ son menores de 70 años y sin embargo en las mujeres el 55,7\% son de 70 y más años.

Al distribuir los casos por sexo y decenios se puede comprobar que las diferencias son, igualmente estadísticamente significativas $(p<0,001)$ para los varones desde el cuarto decenio, no encontrándose diferencias en el grupo etario del noveno decenio (Figura 1).

La histología más frecuentemente encontrada ha sido la de papilomas y carcinomas de células transicionales con un 94,3\%, seguidas de neoplasias epiteliales $(1,7 \%)$, neoplasias papilares y carcinomas de células escamosas $(1,5 \%)$, adenomas y adenocarcinomas $(0,9 \%)$; el resto no sobrepasan el $0,8 \%$. Por ello las hemos agrupado en: Neoplasias uroteliales, Neoplasias de células escamosas, Neoplasias glandulares, Otras neoplasias y Desconocido (Tabla 2).

Las Neoplasias Uroteliales son las más frecuentes con un total de 4.198 casos $(94,3 \%)$
Tabla 1. $N^{o}$ de casos por quinquenio.

\begin{tabular}{|lcc|}
\hline \multicolumn{1}{|c}{ Quinquenios } & Frecuencia & $\mathbf{\%}$ \\
\hline $1990-1994$ & 1.128 & 25,3 \\
$1995-1999$ & 1.831 & 41,1 \\
$2000-2004$ & 1.493 & 33,5 \\
\hline Total & 4.452 & 100 \\
\hline
\end{tabular}

Tabla 2. Distribución del cáncer de vejiga por grupos histológicos.

\begin{tabular}{|lr|}
\hline \multicolumn{1}{|c|}{ Grupo } & \multicolumn{1}{c|}{$\%$} \\
\hline Neoplasias uroteliales & 94,3 \\
Neoplasia de células escamosas & 3,2 \\
Neoplasias glandulares & 0,9 \\
Otros & 0,5 \\
Desconocido & 1,0 \\
\hline Total & 100 \\
\hline
\end{tabular}

\section{Histología y edad}

En ambos grupos de edad, las neoplasias uroteliales son las más frecuentes ( $>90 \%$ de los casos). Si bién se han encontrado diferencias estadísticamente significativas $(p<0,001)$ en la distribución de los grupos histológicos en menores y mayores de 70 años. De los 4.198 casos con neoplasias uroteliales el 53,6\% son sujetos con menos de 70 años y el 46,4\% de 70 o más años. Destacar que en los casos en los que desconocemos la histología el 78\% tiene 70 o más años (Figura 2). La distribución de los tumores por décadas se comprueba como es a partir de la sexta década cuando se incrementa la frecuencia de presentación de los tumores uroteliales y las neoplasias de células escamosas, in-

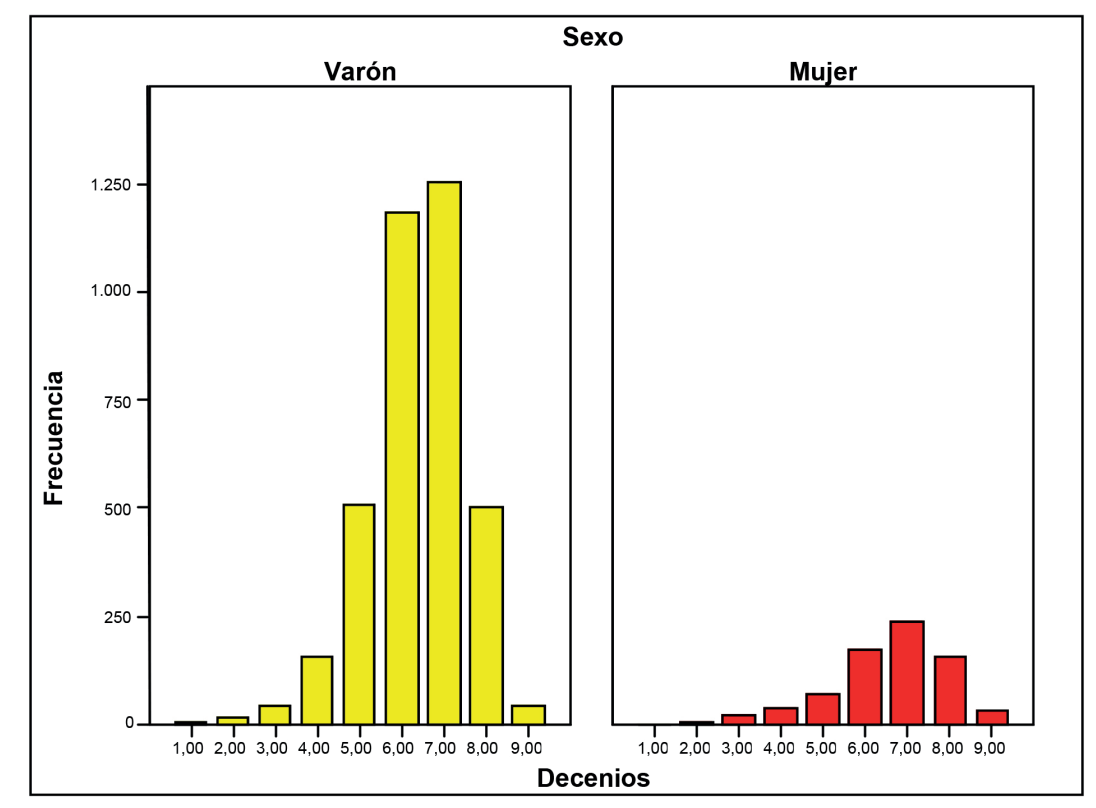

Figura 1. Distribución por sexo y edad en decenios. 


\section{Cáncer de vejiga en hospitales públicos de Madrid}

crementándose a partir de la quinta década el conjunto de presentaciones histológicas que hemos etiquetado como "otros", pero en cualquier caso los tumores uroteliales están presentes en todas las décadas estudiadas (Figura 2).

\section{Histologías agrupadas y sexo}

Las neoplasias uroteliales se han distribuido practicamente igual, $94 \%$ en varones vs $93,9 \%$ en mujeres, y algo parecido ha sucedido con el resto de histologías: Neoplasia de células escamosas $3,3 \%$ vs 3,1\%; Neoplasias glandulares $1 \%$ y $0,4 \%$ y otras histologías $0,5 \%$ y $0,7 \%$ para varones y mujeres respectivamente.

La extensión más frecuentemente encontrada se ha correspondido con el tumor localizado $83,5 \%$, y el menos habitual ha sido el "in situ" $0,7 \%$, por ello, y dado que el gesto terapéutico es idéntico, hemos agrupado los tumores localizados con los “in situ” (Tabla 3).

Tabla 3. Extensión tumoral.

\begin{tabular}{|lc|}
\hline Extensión tumoral & $\mathbf{\%}$ \\
\hline in situ / localizado & 84,2 \\
Regional & 10,6 \\
Diseminado & 4,9 \\
Total & 99,6 \\
Perdidos Sistema & 0,4 \\
\hline Total & 100 \\
\hline
\end{tabular}

\section{Extensión tumoral e histologías}

En todos los grupos histológicos la extensión in situ/ localizada es la más frecuente al diagnóstico, si bien existen diferencias estadísticamente significativas $(\mathrm{p}<0,001)$. En las neoplasias uroteliales el $85,7 \%$ de los casos son in situ/localizados y solo un $4,1 \%$ son diseminados; mientras que en las neoplasias de células escamosas y las neoplasias glandulares la extensión in situ/ localizada es del $61,5 \%$ y $63,2 \%$ de los casos respectivamente; y en ambos aproximadamente el $18 \%$ de los casos son diseminados. Conviene comentar que en los casos del grupo "otros" y de desconocidos, la valoración debe hacerse con cierta cautela dado el número de tumores que se han estudiado.

\section{Extensión tumoral y sexo}

Se han encontrado diferencias estadísticamente significativas $(\mathrm{p}<0,05)$ entre el sexo y la extensión del tumor al diagnóstico. Mientras que en las mujeres el $86.7 \%$ de los casos han sido in situ/localizados, en los hombres han sido el 84,1\%. Los tumores con extensión regional son más frecuentes en los hombres $(11,1 \%$ vs $7,9 \%)$ y los diseminados en ambos sexos son el $5 \%$ aproximadamente.

\section{Localización tumoral}

El 63,3\% de los casos están localizados en vejiga urinaria sine alter indicatio (SAI); en el resto de los casos las localizaciones anatómicas del tumor eran muy diversas (Tabla 4).

Tabla 4. Distribución de la localización tumoral.

\begin{tabular}{|lcc|}
\hline Localización anatómica & Frecuencia & $\mathbf{\%}$ \\
\hline Trígono vesical & 109 & 2,4 \\
Cúpula vesical & 65 & 1,5 \\
Pared lateral de la vejiga & 917 & 20,6 \\
Pared anterior de la vejiga & 20 & 0,4 \\
Pared posterior de la vejiga & 85 & 1,9 \\
Cuello de la vejiga & 61 & 1,4 \\
Orificio ureteral & 47 & 1,1 \\
Lesión de sitios contiguos de la vejiga & 315 & 7,1 \\
Vejiga urinaria, SAI * & 2.833 & 63,6 \\
\hline Total & 4.452 & 100 \\
\hline
\end{tabular}

*= Sine alter indicatio

Es decir, el 4,9\% se localizan en trígono, cuello vesical y orificio ureteral y el $95,1 \%$ en el resto del órgano.

No se han detectado diferencias estadísticamente significativas entre las localizaciones del tumor en la vejiga y la edad agrupada en menores de 70 años y 70 o más años.

Es evidente el predominio entre los varones, con respecto a las mujeres y a la localización "resto", (96\% vs 92\%), siendo las diferencias estadísticamente significativas $(\mathrm{p}<0,001)$.

No hemos encontrado diferencias estadísticamente significativas $(p>0,05)$ entre la localización del tumor y los grupos histológicos; en todos ellos más del 95\% de los casos se localizan en "localización resto".

No se han detectado diferencias estadísticamente significativa entre la localización del tumor y la extensión tumoral ( $\mathrm{p}>$ $0,05)$. Los tumores localizados en trígono, cuello vesical y orificio ureteral sólo llegan al 5\% en los in situ/ localizado, 4\% en los regionales y un 3,2\% en los diseminados (Tabla 5). 
En nuestra serie, de un total de 4.452 pacientes se han presentado 198 tumores primarios múltiples (4\%) que cumplían todos los requisitos de la IARC.

Los 198 tumores primarios múltiples se han repartido en las diferentes histologías del siguiente modo: Uroteliales 90,4\%, Neoplasias de células escamosas 4,5\%, Neoplasias glandulares 1 $\%$, Otros $1 \%$ y un $3 \%$ de desconocidos, habiéndose encontrado diferencias estadísticamente significativas $(\mathrm{p}<0,05)$.

Tabla 5. Localización y extensión tumoral.

\begin{tabular}{|lcc|}
\hline Extensión tumoral & \multicolumn{2}{c|}{ Localización (\%) } \\
\hline & $\begin{array}{c}\text { Trígono, cuello vesical y } \\
\text { orificio ureteral }\end{array}$ & Resto \\
\hline In situ /localizado & 5,1 & 94,9 \\
Regional & 4,0 & 96,0 \\
Diseminado & 3,2 & 96,8 \\
\hline
\end{tabular}

Tabla 6. Tratamientos principales (Recibidos por más del 1\% de los pacientes).

\begin{tabular}{|lc|}
\hline Tratamiento & $\mathbf{\%}$ \\
\hline Sin tratamiento & 1,2 \\
Cirugía & 37,8 \\
Tratamiento paliativo & 2,7 \\
Cirugía -Radioterapia & 6,2 \\
Cirugía -Quimioterapia & 38,1 \\
Cirugía -Inmunoterapia & 6,0 \\
Otros & 78 \\
\hline
\end{tabular}

Tabla 7. Distribución de los tratamientos por grupos de edad.

\begin{tabular}{|lcc|}
\hline Tratamientos & \multicolumn{2}{c|}{ Edad (\%) } \\
\hline & $<70$ años & $\geq 70$ años \\
\cline { 2 - 3 } Cirugía & 50,4 & 49,6 \\
Cirugía-radioterapia & 25,8 & 74,2 \\
Cirugía-quimioterapia & 59,3 & 40,7 \\
Cirugía-inmunoterapia & 61,1 & 38,9 \\
Paliativos & 30,5 & 69,5 \\
Otros tratamientos & 63,6 & 36,4 \\
\hline Total & 2.366 & 2.076 \\
\hline
\end{tabular}

\section{Tratamiento}

Los tratamientos realizados, solos o asociados, han sido más de 40; destacando el 37,8\% a los que se le ha realizado sólo tratamiento quirúrgico y a continuación, con una cifra muy parecida a los que se les ha efectuado una cirugía y quimioterapia $(38,1 \%)$; el resto de los gestos terapéuticos se distribuyen con un porcentaje sensiblemente inferior (Tabla 6). El término sin tratamiento se refiere a que estos pacientes no han recibido tratamiento en el mismo centro que se realiza el diagnóstico. Al agrupar los tratamientos en mono y multidisciplinares, incluyéndose los no tratados, un $43,3 \%$ ha recibido un tratamiento monodisciplinar y más del $50 \%$ ha recibido un tratamiento multidiscipliar (Tabla 6).

Se han detectado diferencias estadísticamente significativas entre ambos grupos etarios $(\mathrm{p}<0,001)$. La cirugía más radioterapia se ha indicado con más frecuencia entre los mayores de 70 años, sucediendo lo mismo con los pacientes que han recibido tratamientos paliativos, y observándose lo contrario con el resto de actitudes terapéuticas, con respecto a la edad (Tabla 7).

Destacar que la cirugía como único tratamiento tiene una frecuencia similar en ambos grupos de edad.

Las diferencias encontradas por sexo no se han debido al azar $(p<0,001)$, mientras que en las mujeres el tratamiento más frecuente es la cirugía $(41,7 \%)$, en los varones es la cirugía - quimioterapia $(38,6 \%)$ el gesto terapéutico más utilizado.

El 37,8\% de las neoplasias uroteliales han recibido sólo un tratamiento quirúrgico, un $1,9 \%$ tratamiento paliativo, un $6,2 \%$ un tratamiento multidisciplinar consistente en cirugía más radioterapia y por último la combinación de cirugía más quimioterapia se le administró en el 39,6\%. En cuanto a las neoplasias de células escamosas la pauta terapéutica fue muy similar desde un punto cuantitativo, excepto en el caso de los tratamientos paliativos que llegan al $10 \%$. En el resto de presentaciones histológicas el orden de mayor a menor fue idéntico (cirugía, cirugía más quimioterapia). El resto de actuaciones terapéuticas no las hemos considerado por su escaso numero (Tabla 8). Las diferencias encontradas han alcanzado la significación estadística con un $\mathrm{p}<0,001$.

Se han encontrado diferencias estadísticamente significativas $(\mathrm{p}<0,05)$ entre la actitud terapéutica y la extensión tumoral. A los tumores in situ / localizado se les ha tratado quirúrgicamente en el $38,4 \%$ de las ocasiones, en tanto que a los tumores con extensión regional y diseminada han recibido este tratamiento en el $41,8 \%$ y $22,3 \%$ respectivamente. El tratamiento combinado de cirugía-quimioterapia, ha sido utilizado muy frecuentemente; así

Tabla 8. Distribución de los tratamientos por histologías (\%).

\begin{tabular}{|lccccc|}
\hline Tratamientos & $\begin{array}{c}\text { Neoplasias } \\
\text { Uroteliales }\end{array}$ & $\begin{array}{c}\text { Neoplasia cél. } \\
\text { escamosas }\end{array}$ & $\begin{array}{c}\text { Neoplasias } \\
\text { glandulares }\end{array}$ & Otros & Desconocido \\
\hline Cirugía & 37,8 & 44,4 & 42,5 & 50,0 & 13,0 \\
Paliativo & 1,9 & 10,4 & 0 & 4,2 & 50,0 \\
Cirugía- radioterapia & 6,2 & 7,6 & 0 & 12,5 & 0 \\
Cirugía- quimioterapia & 39,6 & 15,3 & 22,5 & 12,5 & 8,7 \\
Cirugía- inmunoterapia & 6,2 & 2,1 & 0 & 0 & 6,5 \\
Otros & 7,3 & 16,0 & 27,5 & 20,8 & 6,5 \\
\hline Total & 100 & 100 & $100 \%$ & $100 \%$ & 100 \\
\hline
\end{tabular}


Tabla 9. Distribución por edades, nuestra serie vs NCDB (\%).

\begin{tabular}{|lcccccccc|}
\hline & $\mathbf{0 - 1 4}$ & $\mathbf{1 5 - 2 9}$ & $\mathbf{3 0 - 3 9}$ & $\mathbf{4 0 - 4 9}$ & $\mathbf{5 0 - 5 9}$ & $\mathbf{6 0 - 6 9}$ & $\mathbf{7 0 - 7 9}$ & $\mathbf{2 8 0}$ \\
\hline Nuestra serie & 0,1 & 0,6 & 1,3 & 4,4 & 13,0 & 30,50 & 33,50 & 16,5 \\
NCDB & & 0,22 & 0,83 & 3,99 & 12,54 & 23,09 & 31,62 & 27,00 \\
\hline
\end{tabular}

se ha empleado en el $41,1 \%, 27,1 \%$ y $14 \%$ para las extensiones in situ/localizado, regional y diseminado respectivamente. Los tratamientos paliativos han dominado $(31,2 \%)$ para la extensión diseminada.

Todas las actitudes terapéuticas se han dirigido sobre el conjunto que hemos denominado "resto" de localizaciones anatómicas, encontrándose diferencias estadísticamente significativas $(\mathrm{p}<0,05)$. En este caso hay que evaluar con cautela estos resultados porque el número total de "resto" era sensiblemente superior, 4.225 frente a 217 de las localizaciones de trígono, cuello vesical y orificio ureteral. Del total de tumores localizados en trígono, cuello vesical y orificio ureteral el $40,6 \%$ de los casos han recibido cirugía-quimioterapia y el $34,1 \%$ cirugía, mientras que los de localización "otros", la cirugía sola y asociada a quimioterapia ha sido igual de frecuente $(38,1 \%)$.

\section{DISCUSIÓN}

La tendencia alcista encontrada en el período de estudio, coincide con la American Cancer Society (ACS), que pasa de 70.980 nuevos casos en 2009 a 72.570 en $2013^{5}$. La edad media de nuestra serie coincide prácticamente con Megwalu et al., 69 vs 71 años, y en nuestro medio coincide plenamente con la edad encontrada por Serrano et al., (68 años $)^{6,7}$. La distribución por edades se ha agrupado entre la sexta y séptima década, coincidiendo parcialmente con la serie del $\mathrm{NCDB}^{8}$ para el grupo etario de 70 y más años (33,5\% vs 31,6\%) (Tabla 9). No hemos observado un pico importante en la octava década, especialmente a los 85 años, como se publica en una serie de California, que analizando 92.159 casos detectan un incremento importante en el grupo etario de 85 años; los autores no encuentran una explicación lógica para este hallazgo y encontramos una práctica coincidencia con la serie del SEER ${ }^{9,10}$. Entre los menores de 75 años el riesgo de desarrollar una neoplasia de vejiga es del $2 \%$, de este porcentaje el $4 \%$ se refiere a los varones y el $0,5 \%$ a mujeres ${ }^{11}$.

La distribución por sexos encontrada en nuestra serie ha sido mayor entre los varones, un $83,4 \%$ vs $16,6 \%$ en mujeres, lo que coincide en líneas generales con el $\mathrm{NCDB}(74,2 \%$ para varones vs $25,28 \%$ en mujeres $)^{8}$. En cuanto a la razón de sexo de nuestro estudio ha sido sensiblemente superior a la publicada por la OMS, (3:1 vs 5:1) $)^{12}$, siendo mayor en Murcia (8:1), Granada $(8: 1)$, Gerona (6:1), Cuenca $(6: 1)$, y Canarias $(7: 1)^{13}$.

Estas diferencias podrían obedecer al diferente arraigo del hábito tabáquico entre sexos en nuestro País ${ }^{14}$, aunque probablemente obedezca a la presencia de receptores androgénicos en los varones ${ }^{15-17}$.

El carcinoma in situ no se debe considerar en sentido estricto como un cáncer y así se comenta para las diferentes localizaciones anatómicas, sin embargo, la IARC hace dos excepciones a la regla, de una parte para los carcinomas in situ de la vejiga y otra
Tabla 10. Histología de nuestra serie vs $N C D B$.

\begin{tabular}{|c|c|c|c|}
\hline & Tumores & Casos & $\%$ \\
\hline \multirow[t]{5}{*}{ Nuestra serie } & Neoplasias Uroteliales & 4.198 & 94,30 \\
\hline & N. células escamosas & 144 & 3,20 \\
\hline & N. células glandulares & 40 & 0,90 \\
\hline & Otros & 24 & 0,50 \\
\hline & Desconocidos & 46 & 1,00 \\
\hline \multirow[t]{3}{*}{ NCDB } & C. células transicionales & 11.492 & 28,28 \\
\hline & C. papilar & 26.412 & 65,01 \\
\hline & Otros & 2.726 & 6,71 \\
\hline
\end{tabular}

Tabla 11. Neoplasias uroteliales por sexo, nuestro estudio vs NCDB.

\begin{tabular}{|lcc|}
\hline & NCDB & Nuestro estudio \\
\hline Varón & $94,10 \%$ & $94,4 \%$ \\
Mujer & $90,8 \%$ & $93,9 \%$ \\
\hline
\end{tabular}

Tabla 12. Comparación extensión tumoral (\%).

\begin{tabular}{|ccc|}
\hline Extensión tumoral & SEER & Nuestro Estudio \\
\hline in situ / localizado & 74,1 & 84,2 \\
Regional & 18,7 & 10,6 \\
Diseminado & 3,7 & 4,9 \\
\hline
\end{tabular}

para lo que se ubican en la mama ${ }^{18}$. La agrupación realizada por nosotros incluye dentro de las neoplasias uroteliales a los carcinomas de células transicionales y a los papilomas de células transicionales. Las histologías más frecuentemente detectadas en el cáncer de vejiga han sido los papilomas y carcinomas de células transicionales $(94,3 \%)$, no discrepando en este punto con lo publicado por el $\mathrm{NCDB}^{8}$ (Tabla 10).

Las histologías más frecuentemente detectadas en el cáncer de vejiga han sido los papilomas y carcinomas de células transicionales $(94,3 \%)$, no discrepando en este punto con lo publicado por $\mathrm{NCDB}^{8}$. Si sumamos los carcinomas papilares y los de células transicionales (37.904 casos), comprobaremos que las diferencias encontradas con respecto a nuestra serie es mínima $(93,2 \%$ vs $94,3 \%)$. La serie americana presenta una frecuencia mayor en el epígrafe de "otros" que el encontrado por nosotros".

La distribución de la neoplasia encontrada, se corresponde, entre los mayores de 70 años con las histologías consideradas más agresivas, lo que coincide con otros autores ${ }^{10,19}$.

Nuestra serie ha presentado resultados muy similares a los del NCDB, referidos a las neoplasias uroteliales y su distribución por sexo. Como se observa en la tabla la frecuencia de presentación en hombres es la misma que la del NCDB y algo superior en nuestra serie para las mujeres (Tabla 11$)^{8}$. 
Es cierto por otra parte que en las publicaciones del National Cancer Institute se puede observar la misma clasificación realizada en la extensión tumoral que hemos empleado nosotros ${ }^{8}$. Igualmente en publicaciones recientes se emplea la misma clasificación que nosotros ${ }^{10,20}$. En la serie correspondiente al SEER (17 areas) para el periodo 2000-2005 podemos comprobar que el tumor in situ/localizado es un $10 \%$ menos frecuente que en nuestra serie $(74,1 \%$ vs $84,2 \%$ ), los tumores con extensión regional un $8 \%$ más frecuentes que nosotros y los diseminados $1 \%$ menos frecuente (Tabla12) ${ }^{21}$.

En la serie correspondiente a 17 áreas de EEUU publicadas por el SEER encontramos diferencias en relación con la extensión tumoral en ambas sexos similares a las encontradas en la extensión tumoral para ambos. En nuestra serie los tumores in situ/ localizado son mas frecuentes en mujeres mientras que en los datos del SEER es al contrario y lo mismo ocurre con los regionales. Los tumores diseminados en ambas series son muy similares ${ }^{21}$.

El hecho de que la extensión tumoral "in situ" y localizada se asocie de un modo importante con los tumores uroteliales es lógico y coherente; la extensión es la más dominante en nuestra serie, siendo igualmente muy frecuente en las neoplasias uroteliales. En el caso del carcinoma de células transicionales (neoplasias uroteliales) se ha comprobado que entre el 15 y el $30 \%$ van a proseguir a extensiones más peligrosas para el paciente.

Encontramos una discrepancia importante con la serie del $\mathrm{NCDB}$, en cuanto a la cirugía se refiere; en la serie americana un tratamiento sólo quirúrgico se les ha indicado al 72,90\% vs un $37,9 \%$, sin embargo la resección transureteral no se especifica si ha sido o no asociada a quimioterapia intravesical; en nuestra serie, si ahora unimos cirugía con quimioterapia, se alcanza el $76 \%$, dentro de este porcentaje se ha contemplado no sólo la cirugía como acto quirúrgico aislado sino la asociación con la quimioterapia, local o sistémica. En el caso de la unión de la cirugía con la quimioterapia la indicación en nuestros pacientes ha sido sensiblemente mayor que la publicada en USA $(38,1 \%$ vs $9,08 \%)$, este discrepancia pudiera obedecer a que no han hecho referencia nada más que a la quimioterapia sistémica. Prácticamente coincidimos con la combinación de cirugía e inmunoterapia $(6 \%$ en nuestra serie vs 7,24\%) y en otras terapias $(7,8 \% \text { vs } 6,64 \%)^{8}$.

El tratamiento quirúrgico, bien sólo o en combinación con otros, es el gesto terapéutico que se indica en más del 90\% de los casos, según publica la Sociedad americana del cáncer ${ }^{22}$. Si tenemos en cuenta ahora que el 55\% de nuestra población es menor de setenta años, es este grupo etario el que más se beneficia del tratamiento menos agresivo; sin embargo en otros estudios, tomando una población de mayores y menores de 75 años con carcinoma vesical infiltrante, se cuestionan si la cistectomía radical más la linfadenectomía estaba o no justificada ${ }^{23}$. Los pacientes mayores de 70 años suelen padecer procesos más agresivos y con mayor porcentaje de progresión, por ello no es de extrañar que los gestos terapéuticos sean más agresivos. En nuestro caso sucede algo parecido, los pacientes de 70 y más años han recibido más tratamientos paliativos, lo que se justifica por una mayor agresividad del tumor. Igualmente son estos pacientes los que han recibido con más frecuencia cirugía más radioterapia ${ }^{7}$.
Encontramos una virtual coincidencia con los datos publicados por el NCDB, en lo referente al tratamiento sólo quirúrgico, apreciándose un decalaje de un $10 \%$ en sólo cirugía y en la combinación de cirugía más quimioterapia (Tabla 13), discrepando para el resto de las situaciones terapéuticas. En cualquier caso hay que comentar que en nuestra serie sí se recoge la posibilidad de tratamiento paliativo, no figurando esta posibilidad en la serie americana $^{8}$.

Nuestra serie coincide, en líneas generales, con la combinación de cirugía más inmunoterapia, (por ser éste un gesto terapéutico universalizado) discrepando en el resto de las actitudes terapéuticas. De nuevo comentamos que la serie americana no contempla los tratamientos paliativos y además desagrega los casos que no han recibido como primer tratamiento la radioterapia (Tabla 14) $)^{8}$.

Asumiendo las diferencias ya detectadas en cuanto a los tratamientos y a la clasificación histológica, al sumar los tratamientos sólo de cirugía dirigidos a los carcinomas de células transicionales y papilar transicional, nuestros resultados son muy similares a los suyos ( $74 \%$ y nosotros $77,4 \%$ ).

En cualquier caso, el $90 \%$ de los tratamientos en el cáncer localizado consiste en: cirugía sola o combinada con quimioterapia, radioterapia o inmunoterapia ${ }^{8}$.

Resulta muy dificultoso discutir la asociación entre el tratamiento y la extensión del tumor, dado que los carcinomas en situación de localizados reciben un tratamiento diferente, desde una resección transuretral sóla o asociada con quimioterapia / inmunoterapia hasta llegar una cistectomía radical sóla o asociada a radioterapia y/o quimioterapia sistémica.

En el caso de los regionales se emplea un tratamiento standard consistente en la cistectomía radical precedida o no de radio o quimioterapia (tratamiento neoadyuvante).

Creemos aportar un conocimiento importante al clinico que diagnostica y trata a los pacientes procedentes de una población que forma parte de la que se ha hecho este etudio. Y por tanto puede orientarse con ese paciente a la hora del diagnóstico, estando al día en la praxis seguida en los tratamientos del carcinoma de vejiga en Madrid.

Queremos reseñar las limitaciones del estudio; al tratarse de un registro hospitalario, desconocemos algunos aspectos que nos hubiera gustado conocer. Hubiera sido deseable tener los datos de los tratamientos desagregados; si se ha hecho radiote- 


\section{Cáncer de vejiga en hospitales públicos de Madrid}

rapia, de que modo y como se ha pautado. Si se ha tratado con quimioterapia, con que y a que dosis. Y lo mismo diriamos del tratamiento quirúrgico.

\section{BIBLIOGRAFÍA}

1. http://European cancer observatory eco.iarc.fr

2. http://Centro nacional de epidemiología cne.isciii.es/mapserver/Mapcons. php5

3. Gloeckler Ries LA., Horner MJG., y Young JL., Jr En Ries Lag, Young JL, Keel GE, Eisner MP, Lin YD, Horner M-J (editors).Introduction. SEER Survival Monograph: Cancer Survival Among Adults: U.S. SEER Program, 1988-2001, Patient and Tumor Characteristics. National Cancer Institute, SEER Program, NIH Pub. No. 07-6215, Bethesda, MD, 2007. Pág 3-8.

4. Curado MP ON, Ries LAG, Sriplung H, Young J, et al. International rules for multiple primary cancers. International Classification Diseases - Oncology. 3 th ed. 2004 Lyon : IARC.WHO.ENCR.

5. American Cancer Society. Cancer Facts y Figures. Atlanta: American Cancer Society; 2.009-2013.

6. Megwalu II, Vlahiotis A, Radwan M, Piccirillo JF, Kibel AS. Prognostic impact of comorbidity in patients with bladder cancer. Eur Urol 2008 Mar;53(3):581-9.

7. Serrano FP, Gil MP, Gil Sanz MJ, Felipo F, Allue LM, Rioja Sanz LA. Are there any differences between the characteristics and the behavior of bladder cancer according to the age of presentation? Our experience. Actas Urol Esp 2007 Sep;31(8):825-30.

8. Flesher NE,Herr HW, Stewart AK, Murphy GP, Mettlin C, Menck HR. TheNational Cancer Data Base Report on Bladder Carcinoma. Cancer 1996;78:1505-13.

9. Seer cancer statistics review 1975-2010-seer website seer.cancer.gov/csr

10. Schultzel M, Saltzstein SL, Downs TM, Shimasaki S, Sanders C, Sadler GR. Late age (85 years or older) peak incidence of bladder cancer. J Urol 2008 Apr;179(4):1302-5.
11. Kirkali Z, Chan T, Manoharan M, Algaba F, Busch C, Cheng L, et al. Bladder cancer: epidemiology, staging and grading, and diagnosis. Urology 2005 Dec;66(6 Suppl 1):4-34.

12. World Cancer Report. Edited by Bernard W. Stewart Paul Kleihues IARC Press Lyon 2003.

13. Parkin DW WSF Jeale. Cancer incidente Lyon, France: IARC 1997.2008: 423-43.

14. Infante C, Rubio-Colavida JM. La prevalencia del consumo de tabaco en España. Adicciones 2.004;16(supl.2): 59-73.

15. Hartge P, Harvey EB, Linehan WM, et al. Unexplained excess risk of bladder cancer in men. J Natl Cancer Inst 1990;82:1636-40.

16. Hemelt M, Yamamoto H, Cheng KK, Zeegers MPA. The effect of smoking on the male excess of bladder cancer: a meta-analysis and geographical analyses. Int J Cancer 2009;124:412-9.

17. Izumi K, Zheng Y, Li Y, Zaengle J , H Miyamoto . Epidermal growth factor induces bladder cancer cell proliferation through activation of the androgen receptor. Int J Oncol 2012 Nov;41(5):1587-92.

18. Whelan S.L. Classification and coding in Cáncer incidence in five Continents Vol VIII International Agency for Research on Cáncer. Lyon 2.002 pág 36.

19. Shi B, Zhang K, Zhang J, Chen J, Zhang N, Xu Z. Relationship between patient age and superficial transitional cell carcinoma characteristics. Urology 2008 Jun;71(6):1186-90.

20. Nieder AM, Mackinnon JA, Huang Y, Fleming LE, Koniaris LG, Lee DJ. Florida bladder cancer trends 1981 to 2004: minimal progress in decreasing advanced disease. J Urol 2008 Feb;179(2):491-5.

21. Lynch C.F., Davila J.A., y Platz C.L. en Ries LAG, Young JL, Keel GE, Eisner MP, Lin YD, Horner M-J (editors). Cancer of the urinary bladder. SEER Survival monograph: Cáncer survival among adults: U.S. SEER program, 1988-2.001. Patienst and tumor Characteristics. National Cancer Institute, SEER program, NIH. Pub n ${ }^{\circ}$ 07-6215, Cáncer of the bladder cancer Betseda MD.2.007 pág 181-92.

22. American Cancer Society. Cancer Facts \& figures, 2.001 al 2.013

23. Collado Serra A, Solsona Narbón E., Rubio Briones J., Casanova Ramón-Borja J., IborraJuan I., Ricós Torrent JV. ¿Está justificada la cistectomía radical en pacientes mayores de 75 años? Actas Urol Esp 2.008; 32(3): 288-96. 